\title{
A Soft+Rigid Hybrid Exoskeleton Concept in Scissors-Pendulum Mode: A Suit for Human State Sensing and an Exoskeleton for Assistance
}

\author{
Barkan Ugurlu, Merve Acer, Duygun E. Barkana, Ikilem Gocek, Ayse Kucukyilmaz, Yunus Z. Arslan \\ Halil Basturk, Evren Samur, Emre Ugur, Ramazan Unal and Ozkan Bebek
}

\begin{abstract}
In this paper, we present a novel concept that can enable the human aware control of exoskeletons through the integration of a soft suit and a robotic exoskeleton. Unlike the state-of-the-art exoskeleton controllers which mostly rely on lumped human-robot models, the proposed concept makes use of the independent state measurements concerning the human user and the robot. The ability to observe the human state independently is the key factor in this approach. In order to realize such a system from the hardware point of view, we propose a system integration frame that combines a soft suit for human state measurement and a rigid exoskeleton for human assistance. We identify the technological requirements that are necessary for the realization of such a system with a particular emphasis on soft suit integration. We also propose a template model, named scissor pendulum, that may encapsulate the dominant dynamics of the human-robot combined model to synthesize a controller for human state regulation. A series of simulation experiments were conducted to check the controller performance. As a result, satisfactory human state regulation was attained, adequately confirming that the proposed system could potentially improve exoskeleton-aided applications.
\end{abstract}

\section{INTRODUCTION}

A practical method for walking recovery in paraplegia patients is the use of active exoskeleton robots. In this method, the patient is provided with a wearable robotic system that has the capability to move the legs forward. The first generation of exoskeleton systems can only support active $\mathrm{F} / \mathrm{E}$ (Flexion/Extension) movement through the hip and knee joints, while the healthy upper body provides balance using aids such as walkers and crutches [1]-[3].

B. Ugurlu, R. Unal, and Ozkan Bebek are with the Dept. of Mechanical Engineering, Ozyegin University, 34794 Istanbul, Turkey. e-mail: \{barkan.ugurlu, ramazan.unal, ozkan.bebek\}@ozyegin.edu.tr

D. E. Barkana is with the Dept. of Electrical and Electronics Engineering, Yeditepe University, 34755, Istanbul, Turkey. e-mail I: duyguneroldyeditepe.edu.tr

I. Gocek is with the Dept. of Textile Engineering, Istanbul Technical University, 34437, Istanbul, Turkey. e-ma i l : gocek i @ itu.edu.tr

M. Acer is with the Dept. of Mechanical Engineering, Istanbul Technical University, 34437, Istanbul, Turkey. e-mail : acerme itu.edu.tr

A. Kucukyilmaz is with the School of Computer Science, University of Lincoln, LN6 7TS, Lincoln, U.K. e-mail: akucukyilmaz@lincoln.ac.uk

E. Samur is with the Dept. of Mechanical Engineering, Bogazici University, 34342, Istanbul, Turkey. e-mail: evren. samuraboun. edu.tr

E. Ugur is with the Dept. of Computer Engineering, Bogazici University, 34342, Istanbul, Turkey. e-mail: emre.ugur@boun.edu.tr

Y. Z. Arslan is with the Dept. of Mechanical Engineering, Istanbul University, 34320, Istanbul, Turkey. e-mail: yzarslaneistanbul.edu.tr

H. Basturk was with the Dept. of Mechanical Engineering, Bogazici University, 34342, Istanbul, Turkey. He is currently with a private company. e-mail: basturkhalil@gmail.com
Although these robots could not provide full walking support to the patient, the body weight was relatively reflected to the ground, resulting in reduced physiological problems such as pressure sores, bone and muscle loss, lower circulation of blood in the lower trunk, and improved patients' psychological recovery as a result of walking recovery [1]-[5]. However, the functionality of these robots are often argued and certain improvements are required [6]-[9].

Exoskeleton robots can be used effectively to provide paraplegics with walking benefit, but one of the most important problems encountered in the control of these systems is the inability to observe the physical state of the human. Some researchers assume that the robot and human joints coincide exactly, but that the rotation center of the human knee joint is not stable throughout the movement invalidates this hypothesis [10], [11]. Even if there is full overlap with the use of self-aligning mechanisms, data can only be obtained from the knee and hip joints, causing the remaining human joints data to be not retrievable. To provide a possible solution this problem, EMG (electromyography) sensors and goniometers have been used, it is a matter of discussion whether these offer a permanent solution due to their undesirable characteristics, e.g., day-to-day calibration, noise, deployment issues, and fragility [12].

Due to the loss of the sensorimotor control concerning the lower body of the paraplegics, the common control strategy of the exoskeleton robots is to track preplanned joint trajectories with high-gain controllers, e.g., PID. Although such strategies are sufficient for basic walking support, they cannot guarantee a feasible walking performance of the human-robot integrated system [13]. Since the mutual interaction of the human-robot integrated system with the outer environment is not included within the controller, a consistent and global characterization of the walking motion cannot be acquired [14]. In other words, most state-of-theart exoskeletons are controlled similar to a playback device, therefore, users undergo long, exhausting and risky training processes to adapt to the exoskeleton robots [8], [15]-[17].

The inability to fully observe the human physical condition has prevented the implementation of comprehensive controllers that were applied to humanoids [18]-[21]. For instance, simplified and abstracted models, such as an inverted pendulum, have been used for bipedal walking controllers in an effective way [20], [21]. Yet, even such simplified controllers may not be directly adapted to exoskeleton-based walking support due to non-collocation between human and robot states. Hence, we argue that human state measurement 


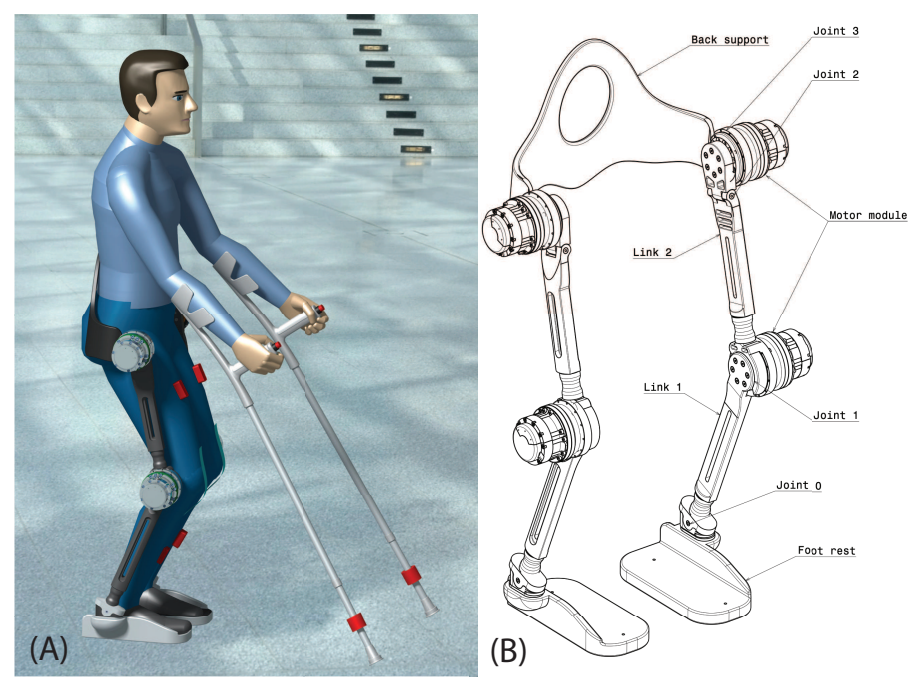

Fig. 1. A) Soft+rigid exoskeleton concept, including a soft suit for human state measurement and a powered exoskeleton for assistance. B) Simplified $\mathrm{CAD}$ data of the lower body exoskeleton that provides assistance through hip and knee F/E joints.

is required to adapt the aforementioned humanoid controllers to exoskeleton-supported walking.

With this aforementioned objective in mind, this paper presents a concept in which a soft suit is integrated into a rigid exoskeleton system to address human state-aware control. To achieve this goal, the required hardware technologies were identified with a particular emphasis on the soft suit for human state measurement. Furthermore, an abstracted model named scissor pendulum was constructed so as to encapsulate the dominant characteristics of a human-robot system. Using this model, a baseline controller was constructed and verified in simulations.

The remainder of the paper is organized as follows. Section II discloses the proposed soft+rigid hybrid exoskeleton concept. Section III explains the scissor pendulum model that is used to represent a human-robot system. Section IV presents the simulation experiments and the paper is concluded in $\mathrm{V}$.

\section{SofT+Rigid Hybrid EXoskeleton CONCEPT}

\section{A. Exoskeleton Structure}

The proposed concept is illustrated in Fig. 1, together with the simplified CAD data of the robot. The system includes a conventional lower body exoskeleton that can provide assistance through hip and knee F/E (Flexion/Extension) joints, in a similar manner to several commercially available devices [1]-[3]. In order to address high-fidelity torquecontrol capability, it is powered via custom-built series elastic actuators [22].

\section{B. Soft Suit}

Recent developments in wearable sensors provide a solution for developing functional, sensory, stretchable suits

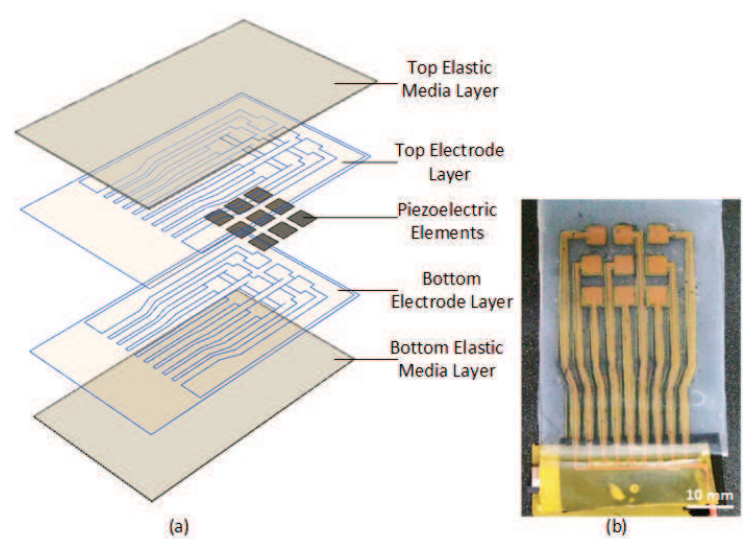

Fig. 2. (a) Layer by layer illustration of $3 \times 3$ matrix formed PZT based tactile sensor. (b) The manufactured tactile sensor [29].

which can be used for human state measurement. 2D layerby-layer manufacturing processes enable us to build lowprofile, embeddable and soft sensors. Besides, the sensor geometry can be customized in accordance with the users' limbs easily. Flexible strain sensors are used for measuring the human joint angles and they will be embedded in an elastic fabric using inkjet printing technologies. They should provide conductivity up to 50-60 degrees. The distributed force sensing elements will be embedded in the sole in order to measure and localize the ground reaction force.

Conductive patterns can be printed using carbon nano powders [23], carbon nanotubes [24], silver nanoparticles [25] and embedded in stretchable clothes or elastomer layers. Inkjet printed stretchable circuits are very promising for the development of these sensors as they can provide high resolution, less complex fabrication, compatibility with the materials for embeddability. While walking, the tension on the flexible strain sensor that is attached to the joints changes and the sensor converts the tension into a change in electrical resistance in relation to the conductive patterns. To measure the resistance change, a Wheatstone bridge circuit is used.

The mapping between the sensor outputs and joint angles must be learned via training. To this end, a certain amount of data collected from a motion capture system is used as a baseline to train necessary functions concerning the aforementioned conversion. While capturing the joint angles, a simplified human skeleton kinematics model can be used [26]. Furthermore, human center of mass can be estimated using the approximate mass distribution over the simplified kinematics model [27], [28].

Force feedback is also a very important feature for the control of exoskeletons. Soft sensors which are able to localize and measure the force are very useful for determining the ground reaction force and center of pressure variations while walking when placed under the feet soles. These sensors are composed of smart materials placed in an array pattern and covered with thin elastomer layers. 9 PZT (Lead Zirconate Titanate, PSI-5H4E) elements are positioned in a $3 \times 3$ matrix form and have 5 layers as shown in Fig. 2. 


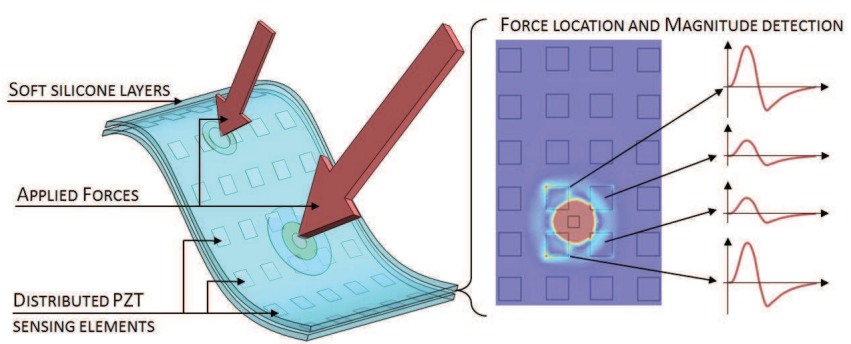

Fig. 3. Wearable, soft piezoelectric based tactile sensor for force location and magnitude detection [30].

The flexible PZT sensor accuracy is $0.578-0.821 \mathrm{~V} / \mathrm{N}$. The electrode paths for each PZT element are designed such that the output signals can be taken individually. First, the copperkapton (Pyralux) sheets are painted and the paint from the patterns which are supposed to be insulated is etched using a laser cutting/etching machine. Second, the copper from these patterns i etched using a solution of hydrogen peroxide $(\mathrm{H} 2 \mathrm{O} 2)$ and hydrochloric acid $(\mathrm{HCl})$. The top and bottom outlines of the electrode patterns are cut using the laser cutting/etching machine. By this way, we have two flexible electrode layers that will connect from the top and the bottom of each PZT element using silver conductive paste (Circuitworks Conductive Epoxy, CW2460). Finally, liquid elastomers (Ecoflex 30) are used to prepare thin $(0.5 \mathrm{~mm})$ elastic layers in order to cover the sensor. The elastomer layers not only protect the PZT elements from the force impacts but also provide the distribution of the forces on the surface which enable to measure the forces continuously on the sensor area. This soft sensor can cover a sole and measure the amplitude and location of the reaction forces while walking; see Fig. 3. This feature will enable to develop the compliant controllers of the human-robot combined system.

\section{Scissor Pendulum Model-based Control}

\section{A. Equations of Motion}

The soft suit embedded within the exoskeleton structure can output all the joint angles and may enable whole-body controllers. As a less computationally-expensive alternative, pendulum-based methods can be used [31]; however, a single inverted pendulum may not represent the dominant characteristics of the system due to non-collocation between human and robot states.

To provide a baseline abstracted model for exoskeletonsupported walking, we propose the scissor pendulum model; see Fig. 4. In this figure, $\theta_{a}, L_{a}, m_{a}, J_{a}, \tau_{a}, F_{a}$ symbolize the angle with the vertical line, pendulum length, mass, approximated inertia, pivot torque, reaction force respectively; $a=r, h$. Subscripts $r$ and $h$ stands for robot and human. The torsional spring-damper couple has the coefficients $k_{i}$ and $b_{i}$.

The model has the following beneficial properties: i) two separate flywheel pendulums with telescopic legs represent human and robot dynamics separately, ii) unlike point mass

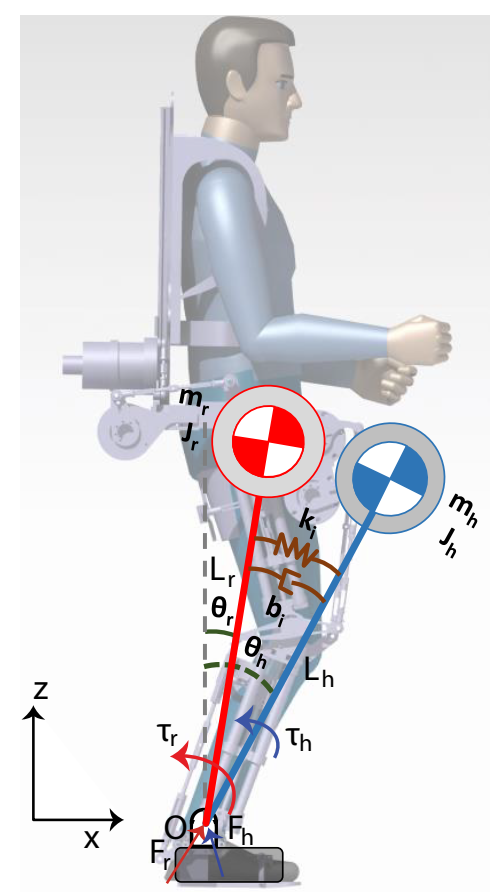

Fig. 4. Scissor pendulum model described on the proposed humanrobot concept. Two flywheel pendulums with a common pivot point, interacting through a spring damper couple can characterize the dominant characteristics concerning the overall balance.

pendulums, it encapsulates the approximated angular momentum via flywheel inertiae [31], iii) the interaction between the human and the robot is represented via a torsional spring-damper couple. In order to derive the equations of motion, Lagrangian mechanics can be used. To this end, the total kinetic energy $\left(T_{s p}\right)$, potential energy $\left(U_{s p}\right)$ and Rayleigh's dissipation $\left(D_{s p}\right)$ can be defined as follows.

$$
\begin{aligned}
T_{s p} & =\frac{1}{2} m_{r}\left(\dot{L}_{r}^{2}+L_{r}^{2} \dot{\theta}_{r}^{2}\right)+\frac{1}{2} J_{r} \dot{\theta}_{r}^{2} \\
& +\frac{1}{2} m_{h}\left(\dot{L}_{h}^{2}+L_{h}^{2} \dot{\theta}_{h}^{2}\right)+\frac{1}{2} J_{h} \dot{\theta}_{h}^{2} \\
U_{s p} & =\frac{1}{2} k_{i}\left(\theta_{h}-\theta_{r}\right)^{2}+m_{r} g L_{r} \cos \theta_{r} \\
& +m_{h} g L_{h} \cos \theta_{h} \\
D_{s p} & =\frac{1}{2} b_{i}\left(\dot{\theta}_{h}-\dot{\theta}_{r}\right)^{2}
\end{aligned}
$$

In (2), $g$ is the gravitational acceleration. Taking the Lagrangian of the system as $\kappa=T_{s p}-U_{s p}$, the following equations are yielded.

$$
\begin{aligned}
F_{r} & =\frac{d}{d t} \frac{\partial \kappa}{\partial \dot{L}_{r}}-\frac{\partial \kappa}{\partial L_{r}}+\frac{\partial D_{s p}}{\partial \dot{L}_{r}} \\
F_{h} & =\frac{d}{d t} \frac{\partial \kappa}{\partial \dot{L}_{h}}-\frac{\partial \kappa}{\partial L_{h}}+\frac{\partial D_{s p}}{\partial \dot{L}_{h}} \\
\tau_{r} & =\frac{d}{d t} \frac{\partial \kappa}{\partial \dot{\theta}_{r}}-\frac{\partial \kappa}{\partial \theta_{r}}+\frac{\partial D_{s p}}{\partial \dot{\theta}_{r}} \\
\tau_{h} & =\frac{d}{d t} \frac{\partial \kappa}{\partial \dot{\theta}_{h}}-\frac{\partial \kappa}{\partial \theta_{h}}+\frac{\partial D_{s p}}{\partial \dot{\theta}_{h}}
\end{aligned}
$$




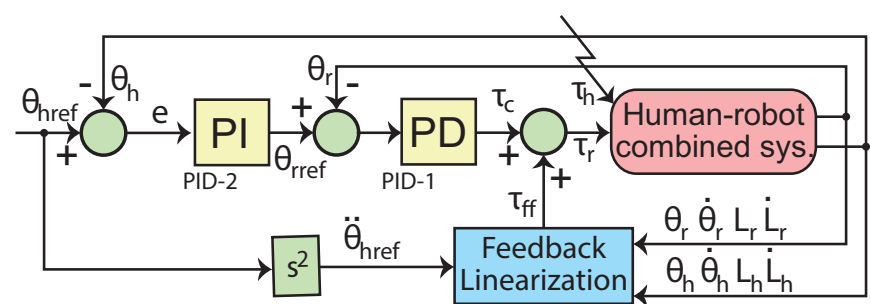

Fig. 5. The cascaded control strategy. The regulation of the human state relies on a faster inner loop control of the robot state. $\tau_{h}$ acts as a source of disturbance, whereas $\tau_{r}$ appears to be the commendable input to the system.

Equations (4) and (5) stand for translational motion whereas (6) and (7) define rotational motion. Assuming sufficient friction between the foot and the floor, we devote our full attention to (6) and (7) as they define the postural balance. By taking the necessary derivation, $\tau_{r}$ and $\tau_{h}$ are obtained as in the following.

$$
\begin{aligned}
\tau_{r} & =J_{o r} \ddot{\theta}_{r}+2 m_{r} L_{r} \dot{L}_{r} \dot{\theta}_{r}-k_{i}\left(\theta_{h}-\theta_{r}\right) \\
& -b_{i}\left(\dot{\theta}_{h}-\dot{\theta}_{r}\right)-m_{r} g L_{r} \sin \theta_{r} \\
\tau_{h} & =J_{o h} \ddot{\theta}_{h}+2 m_{h} L_{h} \dot{L}_{h} \dot{\theta}_{h}+k_{i}\left(\theta_{h}-\theta_{r}\right) \\
& +b_{i}\left(\dot{\theta}_{h}-\dot{\theta}_{r}\right)-m_{h} g L_{h} \sin \theta_{h}
\end{aligned}
$$

In (8) and (9), $J_{o r}=J_{r}+m_{r} L_{r}^{2}$ and $J_{o h}=J_{h}+m_{h} L_{h}^{2}$ symbolize the approximated inertiae that are reflected to pivot $O$.

\section{B. Cascaded Control Strategy}

Scrutinizing (8) and (9), we have two inputs: $\left(\tau_{r}, \tau_{h}\right)$ and two measurable outputs: $\left(\theta_{r}, \theta_{h}\right)$. When considering exoskeleton-supported walking, we would like to control the human state, namely, $\theta_{h}$. Moreover, we have no means of manipulating $\tau_{h}$, the torque generated by the human. Despite the fact that the mathematical model indicates a 2-input 2output system, the application dictates the use of a single input: $\tau_{r}$. Therefore, $\tau_{h}$ can only be considered as a source of disturbance, especially, for the case of paraplegia support as humans may not generate torques voluntarily.

Since the scissor pendulum has a common pivot, (8) and (9) can be summed up:

$$
\begin{aligned}
\tau_{r}+\tau_{h} & =J_{o r} \ddot{\theta}_{r}+2 m_{r} L_{r} \dot{L}_{r} \dot{\theta}_{r}+J_{o h} \ddot{\theta}_{h}+2 m_{h} L_{h} \dot{L}_{h} \dot{\theta}_{h} \\
& -m_{r} g L_{r} \sin \theta_{r}-m_{h} g L_{h} \sin \theta_{h}
\end{aligned}
$$

On the basis of eq. (10), we synthesized a cascaded control system that is somewhat similar to series elastic actuator control [32], with an additional feedback linearization block. Fig. 5 illustrates the proposed controller in which $\theta_{\text {href }}$ is the desired human state, $e$ is the human state error $(e=$ $\left.\theta_{\text {href }}-\theta_{h}\right), \tau_{f f}$ is the feedback linearization torque, $\tau_{c}$ the controller output. The feedback linearization torque accounts for the nonlinear terms in (10) and designed in a similar manner to that of computed torque control method:

$$
\begin{aligned}
\tau_{f f} & =J_{o h} \ddot{\theta}_{h r e f}+2 m_{r} L_{r} \dot{L}_{r} \dot{\theta}_{r}+2 m_{h} L_{h} \dot{L}_{h} \dot{\theta}_{h} \\
& -m_{r} g L_{r} \sin \theta_{r}-m_{h} g L_{h} \sin \theta_{h}
\end{aligned}
$$

As depicted in Fig. 5, $\tau_{r}$ is designated as the sum of feedback linearization torque and the controller output; $\tau_{r}=$ $\tau_{c}+\tau_{f f}$. Therefore, if the parameter uncertainty is negligible, combining (11) and (10) yields the following:

$$
\tau_{c}+\tau_{h}=J_{o r} \ddot{\theta}_{r}-J_{o h} \ddot{e}
$$

where $\tau_{c}$ can be expressed as below.

$$
\begin{aligned}
\tau_{c} & =K_{p 1} K_{p 2} e+K_{p 1} K_{i 2} \int e d t-K_{p 1} \theta_{r} \\
& +K_{d 1} K_{p 2} \dot{e}+K_{d 1} K_{i 2} e-K_{d 1} \dot{\theta}_{r}
\end{aligned}
$$

In (13), $K_{p 2}$ and $K_{i 2}$ represent the outer loop controller (PI) gains while $K_{p 1}$ and $K_{d 1}$ stand for the inner loop controller (PD) gains Finally, plugging (13) into (12), the following error dynamics is obtained.

$$
\begin{aligned}
& K_{p 1} K_{i 2} \int e d t+e\left(K_{p 1} K_{p 2}+K_{d 1} K_{i 2}\right)+K_{d 1} K_{p 2} \dot{e} \\
& +J_{o h} \ddot{e}+\tau_{h}=K_{p 1} \theta_{r}+K_{d 1} \dot{\theta}_{r}+J_{o r} \ddot{\theta}_{r}
\end{aligned}
$$

Using (14), one can construct a transfer function via Laplace transformation, in which $e$ is mapped to $\theta_{r}$ :

$$
\frac{e(s)}{\theta_{r}(s)}=\frac{J_{o r} s^{3}+K_{d 1} s^{2}+K_{p 1} s}{J_{o h} s^{3}+K_{d 1} K_{p 2} s^{2}+K_{a} s+K_{p 1} K_{i 2}}
$$

In (15), $K_{a}=\left(K_{p 1} K_{p 2}+K_{d 1} K_{i 2}\right)$ and $s$ is Laplace variable. With this in mind, the controller gains can be tuned empirically in accordance with the standard rules of linear controller design; stability and non-excitation of unmodeled nonlinear dynamics. For more formal stability analysis, refer to [33].

\section{Simulation Experiments}

Using MATLAB/Simulink, a simulation model comprising the full scissor pendulum dynamics governed by the Eqs. (8) and (9) were constructed. The simulations were run on a PC with Intel Core TM i7 $8550 \mathrm{CPU}$ working at 1.8 $\mathrm{GHz}$ processor and possessing a $16 \mathrm{~GB}$ RAM. As a solver, unconstrained fixed step solver was chosen with a step size of $1 \mathrm{~ms}$. An approximate differentiator with a low pass filter was utilized for time derivative calculations. In all simulation studies, human torque was appeared as a source of step disturbance with an amplitude of $120 \mathrm{Nm}$ and acted at $t=2.4 \mathrm{~s}$. All initial velocity values were assigned as 0 . Model parameters and PID gains are tabulated in Table I.

Simulation results are plotted in Fig. 6 and Fig. 7. In these figures, solid red, solid green, dashed blue and dashed magenta lines respectively indicate measured human state, measured robot state, reference human state, and reference robot state. 


\begin{tabular}{|c|c|}
\hline Parameter Name & Value \\
\hline Human Mass $\left(m_{h}\right)$ & $80 \mathrm{~kg}$ \\
Robot Mass $\left(m_{r}\right)$ & $40 \mathrm{~kg}$ \\
Human Inertia $\left(J_{h}\right)$ & $0.04 \mathrm{kgm}^{2}$ \\
Robot Inertia $\left(J_{r}\right)$ & $0.02 \mathrm{kgm} \mathrm{m}^{2}$ \\
Interaction Spring $\left(k_{i}\right)$ & $800 \mathrm{Nm} / \mathrm{rad}$ \\
Interaction Damper $\left(b_{i}\right)$ & $160 \mathrm{Nms} / \mathrm{rad}$ \\
Initial Human Angle $\left(\theta_{h 0}\right)$ & $-3^{\circ}$ \\
Initial Robot Angle $\left(\theta_{r 0}\right)$ & $2^{\circ}$ \\
Initial Human Pendulum Length $\left(L_{h 0}\right)$ & $0.45 \mathrm{~m}$ \\
Initial Robot Pendulum Length $\left(L_{r 0}\right)$ & $0.33 \mathrm{~m}$ \\
Outer Loop, $K p_{2}$ Gain & 0.5 \\
Outer Loop, $K i_{2}$ Gain & 18 \\
Inner Loop, $K p_{1}$ Gain & 41000 \\
Inner Loop, $K d_{1}$ Gain & 2260 \\
Low Pass Filter Cut-Off Frequency & $50 \mathrm{~Hz}$ \\
Sampling Rate & $1 \mathrm{kHz}$ \\
\hline
\end{tabular}

TABLE I

SIMULATION PARAMETERS
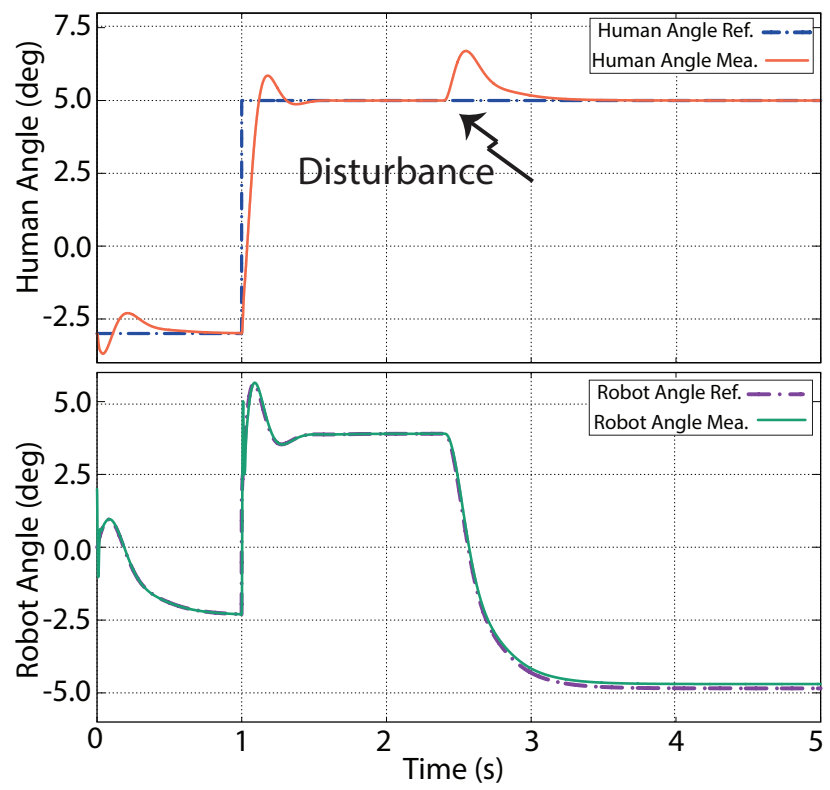

Fig. 6. Step input response, $8^{\circ}$ amplitude.

In Fig. 6, a step input was implemented to pull the human state from initial $-3^{\circ}$ to final $5^{\circ}$. The settling time was observed to be $0.45 s$ with $17 \%$ overshoot. At $t=2.4 s$, the disturbance torque $\tau_{h}$ acted upon the system, causing an overshoot of $34 \%$. The system recovered from this disturbance within $1 \mathrm{~s}$. Finally, the steady-state error was 0.01 millidegrees. The same figure also indicates the reflections on the robot state; in order to ensure the human state regulation, the robot state reference varied in a certain manner. For instance, the robot state reference shifted for about $8.6^{\circ}$ below to continue suppressing the constant disturbance of $120 \mathrm{Nm}$. A steady-state error of $0.15^{\circ}$ was also observed for the robot state tracking.

In Fig. 7, a sine input with a frequency of $0.5 \mathrm{~Hz}$ and peak-to-peak amplitude with $10^{\circ}$ was implemented to check if the controller can handle time-varying inputs. The system quickly recovered and started following the sine input with
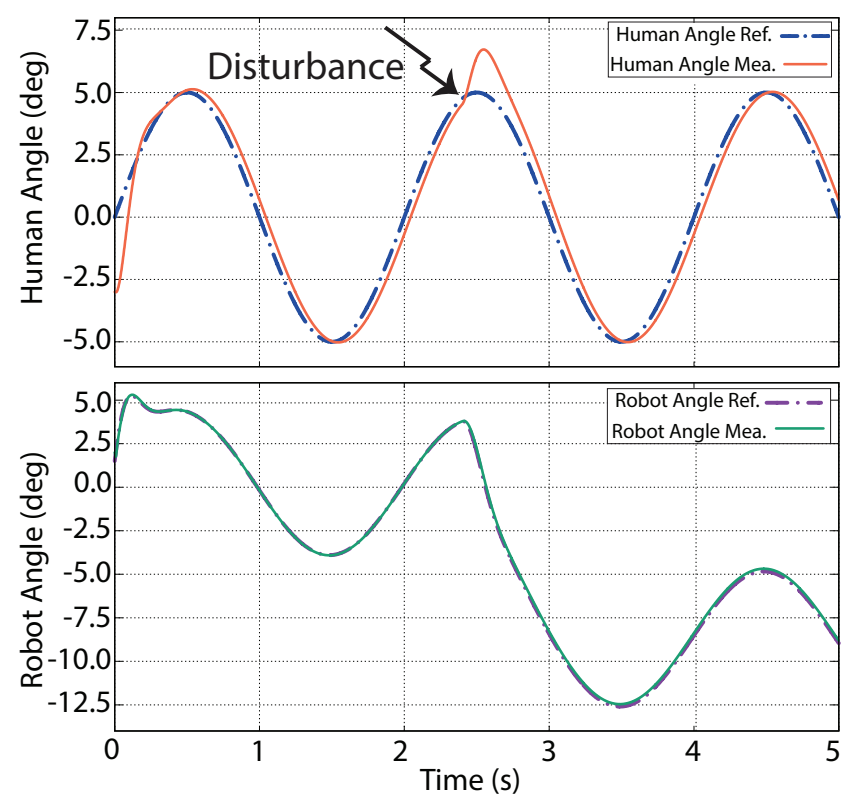

Fig. 7. Sine input response, $10^{\circ}$ peak-to-peak amplitude and $0.5 \mathrm{~Hz}$ frequency.

a delay of $46 \mathrm{~ms}$. At $t=2.4 \mathrm{~s}$, the disturbance torque $\tau_{h}$ acted upon the system, causing the human state to reach $6.7^{\circ}$. Afterward, the controller suppressed the disturbance within $0.5 s$ and continued to track the input signal. The same figure also indicates the reflections on the robot state; the robot state reference varied in a certain manner to attenuate the disturbance. In particular, robot state references shifted for about $8^{\circ}$ below to keep on suppressing the constant disturbance of $120 \mathrm{Nm}$.

\section{CONCLUSION}

This paper presented a novel concept which targeted the human-aware control of exoskeletons by integrating a soft suit and a robotic lower body exoskeleton. In contrast to state-of-the-art exoskeleton controllers that can only process robot state measurements for lumped human-robot models, the proposed concept enabled the use of independent state measurements for the human-robot system. In order to provide such technology from a hardware point of view, a system integration framework was presented with a particular emphasis on the soft suit for human state measurement.

Furthermore, a template model called scissor pendulum was proposed. We argue that the single inverted pendulum models cannot represent the exoskeleton-supported human walking; therefore, the scissor pendulum should be the basic template model. In order to check its effectiveness, a series of simulation experiments were run where a cascaded control strategy with a feedback linearization loop was adopted. As a result, the controller showed a satisfactory convergence and tracking performance both for step and sine inputs. Therefore, the proposed strategy is deemed to be a useful basis and can improve the human state regulation behavior of exoskeletons. We argue that the scissor pendulum model can 
be a basis for more complex and effective control algorithms and may pave the way for advanced human-aware control of lower body exoskeletons.

Currently, the author group is in the process of realizing the proposed hardware. In our future work, we will disclose the details concerning the hardware requirements and our system integration solution to fulfill these requirements. Furthermore, the independent state measurement ability will be exploited to synthesize whole 2-body controllers with human-in-the-loop control abilities [34].

\section{REFERENCES}

[1] A. Tsukahara, Y. Hasegawa, K. Eguchi, and Y. Sankai, "Restoration of gait for spinal cord injury patients using hal with intention estimator for preferable swing speed," IEEE Transactions on Neural Systems and Rehabilitation Engineering, vol. 23, no. 2, pp. 308-318, March 2015.

[2] A. Esquenazi, M. Talaty, A. Packel, and M. Saulino, "The rewalk powered exoskeleton to restore ambulatory function to individuals with thoracic-level motor-complete spinal cord injury," American Journal of Physical Medicine \& Rehabilitation, vol. 91, no. 11, 2012.

[3] K. A. Strausser, T. A. Swift, A. B. Zoss, H. Kazerooni, and B. C. Bennett, "Mobile exoskeleton for spinal cord injury: Development and testing," in ASME 2011 Dynamic Systems and Control Conference and Bath/ASME Symposium on Fluid Power and Motion Control. American Society of Mechanical Engineers, 2011, pp. 419-425.

[4] E. C. Hardin, R. Kobetic, and R. J. Triolo, "Ambulation and spinal cord injury," Physical Medicine and Rehabilitation Clinics of North America, vol. 24, no. 2, pp. 355 - 370, 2013, ambulation in Adults with Central Neurologic Disorders.

[5] A. J. Kozlowski, T. N. Bryce, and M. P. Dijkers, "Time and effort required by persons with spinal cord injury to learn to use a powered exoskeleton for assisted walking," Topics in spinal cord injury rehabilitation, vol. 21, no. 2, pp. 110-121, Spring 2015.

[6] J. C. Moreno, A. J. del Ama, A. de los Reyes-Guzmán, Á. Gil-Agudo, R. Ceres, and J. L. Pons, "Neurorobotic and hybrid management of lower limb motor disorders: a review," Medical \& Biological Engineering \& Computing, vol. 49, no. 10, pp. 1119-1130, aug 2011.

[7] R. E. Cowan, B. J. Fregly, M. L. Boninger, L. Chan, M. M. Rodgers, and D. J. Reinkensmeyer, "Recent trends in assistive technology for mobility," Journal of NeuroEngineering and Rehabilitation, vol. 9, no. 1, p. 20, 2012

[8] J. Wolff, C. Parker, J. Borisoff, W. Mortenson, and J. Mattie, "A survey of stakeholder perspectives on exoskeleton technology," Journal of NeuroEngineering and Rehabilitation, vol. 11, no. 1, p. 169, 2014.

[9] A. J. Young and D. P. Ferris, "State of the art and future directions for lower limb robotic exoskeletons," IEEE Transactions on Neural Systems and Rehabilitation Engineering, vol. 25, no. 2, pp. 171-182, Feb 2017.

[10] A. M. Hollister, S. Jatana, A. K. Singh, W. W. Sullivan, and A. G. Lupichuk, "The axes of rotation of the knee," Clin. Orthop. Relat. Res., no. 290, pp. 259-268, May 1993.

[11] B. Celebi, M. Yalcin, and V. Patoglu, "Assiston-knee: A self-aligning knee exoskeleton," in 2013 IEEE/RSJ International Conference on Intelligent Robots and Systems, Nov 2013, pp. 996-1002.

[12] B. Ugurlu, M. Nishimura, K. Hyodo, M. Kawanishi, and T. Narikiyo, "Proof of concept for robot-aided upper limb rehabilitation using disturbance observers," IEEE Transactions on Human-Machine Systems, vol. 45, no. 1, pp. 110-118, Feb 2015.

[13] B. Ugurlu, H. Oshima, and T. Narikiyo, "Lower body exoskeletonsupported compliant bipedal walking for paraplegics: How to reduce upper body effort?" in 2014 IEEE International Conference on Robotics and Automation, May 2014, pp. 1354-1360.

[14] M. Ishikawa, P. V. Komi, M. J. Grey, V. Lepola, and G.-P. Bruggemann, "Muscle-tendon interaction and elastic energy usage in human walking," Journal of Applied Physiology, vol. 99, no. 2, pp. 603-608, aug 2005.

[15] V. Lajeunesse, C. Vincent, F. Routhier, E. Careau, and F. Michaud, "Exoskeletons' design and usefulness evidence according to a systematic review of lower limb exoskeletons used for functional mobility by people with spinal cord injury," Disability and Rehabilitation: Assistive Technology, vol. 11, no. 7, pp. 535-547, 2016.
[16] I. Benson, K. Hart, D. Tussler, and J. J. van Middendorp, "Exoskeletons' design and usefulness evidence according to a systematic review of lower limb exoskeletons used for functional mobility by people with spinal cord injury," Clinical Rehabilitation, vol. 30, no. 1, pp. 73-84, 2016

[17] A. E. Palermo, J. L. Maher, C. B. Baunsgaard, and M. S. Mash, "Clinician-focused overview of bionic exoskeleton use after spinal cord injury," Topics in Spinal Cord Injury Rehabilitation, vol. 23, no. 3, pp. 234-244, 2017.

[18] S. Feng, X. Xinjilefu, C. G. Atkeson, and J. Kim, "Optimization based controller design and implementation for the atlas robot in the darpa robotics challenge finals," in 2015 IEEE-RAS 15th International Conference on Humanoid Robots (Humanoids), 2015, pp. 1028-1035.

[19] M. DeDonato, V. Dimitrov, R. Du, R. Giovacchini, K. Knoedler, X. Long, F. Polido, M. A. Gennert, T. Padır, S. Feng, H. Moriguchi, E. Whitman, X. Xinjilefu, and C. G. Atkeson, "Human-in-the-loop control of a humanoid robot for disaster response: A report from the DARPA robotics challenge trials," Journal of Field Robotics, vol. 32, no. 2, pp. 275-292, feb 2015.

[20] S. Kajita, F. Kanehiro, K. Kaneko, K. Yokoi, and H. Hirukawa, "The 3d linear inverted pendulum mode: A simple modeling for a biped walking pattern generation," in 2001 IEEE International Conference on Intelligent Robots and Systems, 2001, pp. 239-246.

[21] A. Herdt, N. Perrin, and P.-B. Wieber, "Walking without thinking about it," in 2010 IEEE International Conference on Intelligent Robots and Systems, 2010, pp. 190-195.

[22] M. C. Yildirim, P. Sendur, O. Bilgin, B. Gulek, G. G. Yapici, and B. Ugurlu, "An integrated design approach for a series elastic actuator: Stiffness tuning, fatigue analysis, thermal management," in IEEE-RAS International Conference on Humanoid Robots (Humanoids), 2017, pp. 384-389.

[23] H. M. Bergmann, S. Anastasova-Ivanova, I. Spulber, V. Gulati, P. Georgiou, and A. McGregor, "An attachable clothing sensor system for measuring knee joint angles," IEEE Sensors Journal, vol. 13, no. 10, pp. 4090-4097, oct 2013.

[24] X. Wang, J. Li, H. Song, H. Huang, , and J. Gou, "Highly stretchable and wearable strain sensor based on printable carbon nanotube layers/polydimethylsiloxane composites with adjustable sensitivity," ACS Applied Materials \& Interfaces, vol. 10, no. 8, pp. 7371-7380, 2018.

[25] H. Nakamoto, H. Ootaka, M. Tada, I. Hirata, F. Kobayashi, and F. Kojima, "Stretchable strain sensor with anisotropy and application for joint angle measurement," IEEE Sensors Journal, vol. 16, no. 10, pp. 3572-3579, may 2016.

[26] M. Yong-You, Z. Hui, and J. Shou-Wei, "Realistic modeling and animation of human body based on scanned data," Journal of Computer Science and Technology, vol. 19, no. 4, pp. 529-537, 2004.

[27] P. D. Leva, "Adjustments to zatsiorsky-seluyanov's segment inertia parameters," Journal of Biomechanics, vol. 29, no. 9, pp. 1223-1230, 1996.

[28] R. Dumas, L. Cheze, and J. P. Verriest, "Adjustments to mc-conville et al. and young et al. body segment inertial parame- ters," Journal of Biomechanics, vol. 40, no. 3, pp. 543-553, 2007.

[29] M. Acer and A. F. Yildiz, "Force localization estimation using a designed soft tactile sensor," in The International Symposium on Wearable Robotics (WeRob), 2018.

[30] M. Acer, M. Salerno, K. Agbeviade, and J. Paik, "Development and characterization of silicone embedded distributed piezoelectric sensors for contact detection," Smart Materials and Structures, vol. 24, no. 7, pp. $1-15$, jun 2015

[31] J. Pratt, J. Carff, S. Drakunov, and A. Goswami, "Capture point: A step toward humanoid push recovery,," in IEEE-RAS International Conference on Humanoid Robots (Humanoids), 2006, pp. 200-207.

[32] H. Vallery, R. Ekkelenkamp, H. V. D. Kooij, and M. Buss, "Passive and accurate torque control of series elastic actuators," in 2007 IEEE International Conference on Intelligent Robots and Systems, 2007, pp. 3534-3538.

[33] H. Ito, "A lyapunov approach to cascade interconnection of integral input-to-state stable systems," IEEE Transactions on Automatic Control, vol. 55, no. 3, pp. 702-708, March 2010.

[34] L. Peternel, T. Petric, E. Oztop, and J. Babic, "Teaching robots to cooperate with humans in dynamic manipulation tasks based on multimodal human-in-the-loop approach," Autonomous Robots, vol. 36, no. 1-2, pp. 123-136, 2013. 